\title{
CONDICIONALIDAD REFORZADA A TRAVÉS DE LAS CUBIERTAS VEGETALES EN OLIVAR. OPINIÓN DE LOS AGRICULTORES
}

Colombo, $\mathrm{S}^{\mathrm{a}^{\mathrm{a}}}$., González Dugo M.P ${ }^{\mathrm{b}}$, Blázquez Carrasco ${ }^{\mathrm{b}}$, A., Castro Rodríguez, J $\mathrm{J}^{\mathrm{a}}$.

a IFAPA, Centro Camino de Purchil (Granada, sergio.colombo@juntadeandalucia.es; juan.castro.rodriguez@juntadeandalucia.es). ${ }^{b}$ IFAPA, Centro Camino Alameda del Obispo (Córdoba, mariap.gonzalez.d@juntadeandalucia.es; angel.blazquez@juntadeandalucia.es).

\section{Resumen}

En el cultivo del olivar, en la última década, se ha implantado y extendido el uso de cubiertas vegetales como práctica cultural. El empleo de cubiertas vegetales en olivar se asocia a una multitud de efectos ambientales positivos y por tanto la implementación de estas en los olivares ha sido objeto de ayudas específicas en las medidas agroambientales de diferentes programas de desarrollo rural de Andalucía a partir del año 2000. Sin embargo, según los datos de la encuesta sobre Superficies y Rendimientos Cultivos del Ministerio de Agricultura, Pesca y Alimentación (Esyrce) las cubiertas vegetales en olivar sólo se emplean en alrededor de un $29 \%$ del total de la superficie del olivar andaluz. El objetivo de este trabajo es conocer la opinión de los agricultores con respecto a la puesta de cubiertas vegetales. Por ello se realizan 200 encuestas a olivareros y se determinan los factores de bloqueo y propiciadores a las mismas. Entre ellos, se analiza el impacto de una condicionalidad reforzada que obligue a los agricultores a usar cubiertas vegetales.

\section{Palabras Claves:}

Olivar, Cubierta Vegetal, Opinión Agricultores, Condicionalidad Reforzada, Ecoesquemas

\section{Introducción}

En el cultivo del olivar, en la última década, se ha implantado y extendido el uso de cubiertas vegetales como práctica cultural. En concreto, entre 2009 y 2019 el incremento en la superficie de olivar con cubierta fue de 122705 ha (Esyrce, 2009; Esyrce 2019). A diferencia de otras prácticas culturales, el empleo de cubiertas vegetales en olivar se asocia a una multitud de efectos ambientales positivos. En relación con la erosión, hay abundante bibliografía en España sobre el efecto de protección del suelo debido al uso de cubiertas vegetales, tal como lo atestiguan sucesivos trabajos de Espejo-Pérez et al. (2013), ParrasAlcántara et al. (2016) y Gómez et al. (2017). Respecto al secuestro de carbono y su incorporación al suelo, Nieto et al. (2010), Márquez-García et al. (2013) y Chamizo et al. (2017) ponen de manifiesto que el manejo del suelo influye significativamente en su contenido de carbono orgánico y que el empleo de las cubiertas vegetales es el manejo más indicado para este fin. Paralelamente, hay estudios que demuestran otros efectos positivos del uso de las cubiertas vegetales en olivar, como la mejora de la calidad estética del paisaje (Rodríguez-Entrena et al. 2017), el incremento de la biodiversidad (Castro et al. 2014, Gómez, et al. 2017) y la reducción de la contaminación de los recursos hídricos y otros efectos externos (Colombo et al. 2005). Estos efectos positivos del uso de las cubiertas vegetales son muy demandados por la sociedad, que está dispuesta a pagar a los agricultores para que utilicen cubiertas en sus olivares (Colombo et al. 2006, Rodríguez-Entrena et al. 2014).

Es así evidente que la implantación de las cubiertas vegetales contribuye a la consecución de los objetivos del pacto verde europeo y representa una acción de relevancia en la venidera PAC. Al respecto, en la propuesta del MAPA (2021) sobre condicionalidad reforzada se incluye la obligación (BCAM7) de mantener una cubierta vegetal de anchura mínima de 1 metro en las calles transversales a la línea de máxima pendiente en los cultivos leñosos en pendiente igual o superior al $10 \%$. Además, la promoción de cubiertas vegetales vivas se considera entre las posibles prácticas incluidas en los ecoesquemas en olivar (EU 2021). Por tanto, se espera que las cubiertas vegetales van a tener mayor importancia en la concesión de ayudas de la futura PAC. El objetivo de este trabajo es conocer la opinión de los agricultores con respecto a la implantación de cubiertas vegetales, y poder identificar los factores de bloqueo y propiciadores a las mismas. Los resultados obtenidos se consideran de directa utilidad para el sector del olivar y la administración pública. En el primer caso, porque se muestra al sector la acogida que las cubiertas vegetales han tenido en los olivareros andaluces y que éstas representan un manejo sostenible para el futuro. La administración pública se beneficia de información útil para la definición de políticas agrarias de soporte al olivar. No sólo puede ser de utilidad en la definición de las políticas agroambientales, sino que también 
contribuirá a esclarecer la opinión de los agricultores con respecto a posibles formas de implementación de las futura PAC en términos de condicionalidad reforzada o de aplicación de los ecoesquemas en olivar.

\section{Metodología}

La opinión de los agricultores se obtuvo a través de la realización de 200 encuestas entre los meses de abril y septiembre de 2020 por parte de encuestadores profesionales con amplios conocimientos en el sector del olivar. 57 entrevistas fueron realizadas en la provincia de Jaén, 33 en la provincia de Córdoba, 66 en la provincia de Málaga y 44 en la de Granada. Estas provincias suman el 80\% de la superficie total del olivar andaluz y también son representativas de la heterogeneidad estructural del olivar y de las características edáficas, climáticas y geomorfológicas andaluzas.

El formato de ejecución fue telefónico y/o presencial, según la disponibilidad de los encuestados. En particular, se identificaron agricultores de forma aleatoria directamente "en el campo", y se contactó con cooperativas agrarias para que proporcionaran los contactos telefónicos de olivareros dispuestos a realizar la encuesta. A éstos se le ofrecía la posibilidad de realizar la encuesta por teléfono o en persona.

Las encuestas se estructuraron en cuatro bloques que comprendían: 1) información con respecto a la localización y características estructurales de las explotaciones: 2) información con respecto a las cubiertas vegetales, su manejo y las razones que han llevado al agricultor a su uso o, alternativamente a no implantarlas; 3) satisfacción del agricultor con las cubiertas y análisis de su disponibilidad en mantenerla frente a posible escenarios futuros de política agraria; 4) características sociodemográficas de los entrevistados e información relativa al grado de comprensión y actitud de los entrevistados.

Los entrevistados fueron seleccionados solo si tenían capacidad de gestión de la cubierta en sus explotaciones, es decir si eran agricultores que trabajaban y gestionaban su explotación o, en el caso de no trabajar en ella, que decidían su gestión y manejo. El análisis estadístico de los datos obtenidos se llevó a cabo a través del test chi-cuadrado para la comparación de porcentajes de representatividad de la muestra y del cálculo de las frecuencias individuales y acumuladas para el análisis de las respuestas. Los factores que afectan la probabilidad de que un olivareros tenga cubierta vegetal se han analizado a través de una regresión logística.

\section{Resultados}

La muestra de entrevistados se compone de un $89 \%$ de hombres y un $11 \%$ de mujeres. Un $71 \%$ tiene más de 44 años y entre ellos un $13 \%$ más de 64 . Para un $40 \%$ de la muestra la agricultura es la única fuente de sus ingresos mientras que para el $60 \%$ restante existe una actividad complementaria cuya renta es la principal $(73 \%)$ o secundaria $(27 \%)$. La comparativa de las características de la muestra de agricultores de este trabajo con la descrita por el Plan Director del Olivar de Andalucía (CAPDR, 2015), demuestra que el muestreo empleado es representativo para la población en términos de edad $(\chi 2=0.36, \mathrm{P}=0.86)$, sexo $(\chi 2=$ $1.12, \mathrm{P}=0.36)$ e importancia de la agricultura en los ingresos $(\chi 2=5.16 ; \mathrm{P}=0.08)$.

De los 200 entrevistados la mayoría (63\%) declara tener cubierta vegetal en su finca principal y en casi la totalidad de su explotación. Este porcentaje de agricultores es significativamente superior al observado en la literatura (ESYRCE, 2019), poniendo de manifiesto que en las zonas de estudio el empleo de las cubiertas es mucho más generalizado que lo observado por las encuestas del ESYRCE. No obstante, la diferencia se puede deber a un sesgo de elección de los entrevistados, estando aquellos con cubierta vegetal más dispuestos a participar en la encuesta. También, la diferencia puede deberse a la asunción de considerar cómo agricultores "con cubierta" aquellos que la tienen en su parcela principal o en la mayoría de su explotación, con la posibilidad de tener también parcelas sin cubierta. La tipología preferida de cubierta es la espontánea. Solo un $6 \%$ de los agricultores que tienen implantada cubierta la ha sembrado.

Con respecto a las razones para usar cubiertas vegetales en los olivares, la gran mayoría son de origen medioambiental seguidas por motivos económicos y sociales. Así, el principal motivo es la reducción de la erosión del suelo, seguido por el aumento de la infiltración del agua de lluvia y el incremento de materia orgánica en el suelo. Después de estos aspectos ambientales, los entrevistados citan que el manejo de los olivares con cubiertas es más barato que otros manejos con un empleo continuo de herbicidas o laboreo y que el uso de cubierta es práctica habitual en la zona. Por otro lado, aquellos que han declarado no usar cubierta lo hacen principalmente por motivos técnicos (dificultad percibida con respecto al manejo y problemas del suelo en desarrollar cobertura vegetal). Es llamativo que como factor de bloqueo no aparece como significativa la creencia generalizada entre los olivareros, de que la cubierta vegetal afecta a las producciones del olivo. Así, solo un $10 \%$ de los entrevistados considera que las cubiertas pueden tener impacto en la producción.

Hay que decir que la mayoría de los entrevistados que tienen actualmente cubierta declara que este tipo de manejo del olivar le ha resultado más fácil que otros que empleaban anteriormente, a pesar de que al 
principio les ha costado adaptarse al mismo. Solo un $16 \%$ de los entrevistados declaran que el manejo de cubiertas es más complicado que otros, típicamente el laboreo. Además, el 100\% de los agricultores que tienen cubiertas recomiendan su uso, considerándolo mejor que otro sistema de gestión del suelo. Es más, incluso si "le pagaran" por retirarla, un $80 \%$ declara que no renunciaría a su uso. Los resultados de la regresión logística ponen de manifiesto que la probabilidad de tener cubierta aumenta según los entrevistados tengan un sistema de cultivo ecológico y/o convencional, sean más jóvenes y cuanto más peso represente a actividad agraria en su nivel de ingresos. Por otro lado, variables relacionadas con la estructura de explotación (superficie, presencia de regadío, sistema de cultivo, posibilidad de mecanización), con el género y nivel educativo de los entrevistados no han resultado afectar la probabilidad de tener cubierta vegetal.

A los titulares de explotaciones que no tienen cubiertas vegetales se les preguntó si estarían dispuestos a implantarlas en el caso de que la subvención de la PAC disminuyera por no tenerla. En un segundo paso, entre aquellos que contestaron afirmativamente, se indagó por el porcentaje de reducción de la ayuda PAC que admitirían antes de implantarlas. A aquellos que contestaron negativamente, se les preguntó por el máximo valor de reducción admisible de la ayuda PAC que le forzaría a poner cubiertas vegetales. En la Figura 1 se muestran los resultados. Un 59\% de los entrevistados estaría dispuesto a poner cubierta vegetal frente a una reducción de la PAC por no tenerla; en particular, una reducción de la ayuda de un $20 \%$ llevaría a un $33 \%$ de los que no tienen actualmente a implantarla, mientras que una reducción de un 30\% "forzaría" a un $84 \%$ a implantarla. El $42 \%$ de los titulares declaró que no implantarían cubiertas vegetales incluso frente a fuertes reducciones de las ayudas PAC. En particular, el 93\% de estos estaría dispuesto a renunciar a la subvención de la PAC, considerando que el manejo con cubierta es inviable en sus explotaciones. El $7 \%$ restante cambiarían de opinión frente a una reducción del $30 \%$.

\section{Cuadro 1. Opinión de los entrevistados con relación a la implantación de cubierta vegetal frente a reducciones de la subvención de la PAC.}

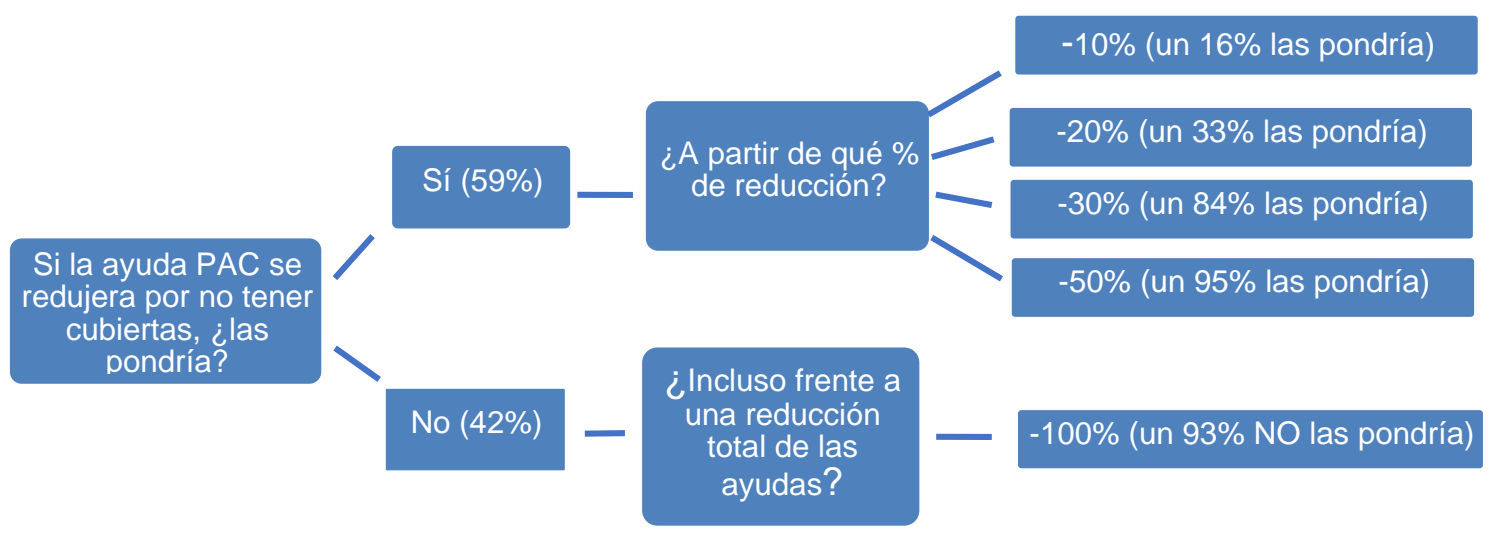

\section{Discusiones}

Los olivareros andaluces tienen una buena opinión con respecto al uso de las cubiertas vegetales. La experiencia tras su uso es muy positiva; aquellos que actualmente tienen implantada la cubierta vegetal la consideran el mejor manejo y aconsejan la adopción. Las razones principales aducidas son que es un sistema más fácil de gestionar y que es más barato, además de numerosas ventajas medioambientales entre las cuales priman la reducción de la erosión del suelo, el aumento de la infiltración del agua y el incremento de materia orgánica en el suelo.

Las percepciones con respecto a la no implantación de la cubierta vegetal ofrecen indicios sobre posibles formas de facilitar la difusión de la adopción de estas. En primer lugar, es necesario proporcionar información a los agricultores con respecto a su manejo. Para paliar el problema de la limitación de maquinaria, se podría proponer una gestión común de las cubiertas a través de las cooperativas agrarias, o a través de la contratación conjunta de los servicios necesarios para su manejo. También, futuras investigaciones deberían analizar qué posibilidades hay de implantación de cubiertas en zonas donde existan limitaciones, bien edáficas por ser suelos muy erosionados y de escaso desarrollo o bien con un amplio historial de uso de herbicidas de preemergencia y un banco de semillas muy enrarecido. También, la formación con respecto a la gestión de las cubiertas vegetales en términos de uso, manejo y beneficios es una herramienta necesaria para garantizar la futura difusión de las cubiertas vegetales y los beneficios aportados por ellas. Solo una minoría de agricultores ha recibido cursos de formación al respecto y prácticamente la totalidad de los entrevistados estaría interesada en participar en cursos de formación. Esta demanda generalizada señala la importancia de que la administración incremente los cursos de formación. 
La formación específica con respecto a diferentes tipologías y estrategias de manejo de cubiertas vegetales, adaptadas a condiciones particulares donde hay dificultad de desarrollo de la vegetación, podría ser una herramienta para incrementar la adopción por parte de aquellos titulares de explotaciones que declaran que nunca pondrían cubierta vegetal debido a esas restricciones edáficas.

El impacto de la política agraria en la adopción y difusión de las cubiertas vegetales puede ser muy significativo. La mayoría de los olivareros que no tienen cubierta la implantaría frente a reducciones de la ayuda de la PAC. La reciente propuesta de la Comisión Europea de incluir las cubiertas vegetales en el listado de prácticas agrarias candidatas formar parte de los futuros ecoesquemas va encaminada en esta dirección y no debería causar mayores rechazos por parte del sector. Sin embargo, hay que analizar con detalle aquellos olivareros donde las condiciones edáficas dificulten el desarrollo de cubiertas vegetales y quizás permitir un periodo de transición que permita la consolidación de las cubiertas. Estudios específicos deberían determinar estas condiciones.

\section{Agradecimientos}

El presente estudio ha sido financiado por el proyecto PR.AVA.AVA2019.031 cofinanciado al 80\% por el Fondo Europeo de Desarrollo Regional, dentro del Programa Operativo FEDER de Andalucía 2014-2020.

\section{Bibliografía}

CAPDR (2015). Plan director del olivar Andaluz. Consejería de Agricultura, Pesca y Desarrollo Rural, Junta de Andalucía.

Castro J.C., Carpio A., Tortosa F.S. (2014) Herbaceous ground cover reduce nest predation in olive groves. Bird Study 61: 537-543.

Chamizo, S., Serrano-Ortiz, P., Lopez-Ballestero, A., Sánchez-Cañete, P., Vicente-Vicente, J., Kowalski, A. (2017). Net ecosystem CO2 exchange in an irrigated Oliver orchard of SE Spain: Influence of weed cover. Agriculture Ecosystem and Environment, 239: 51-64.

Colombo S., Hanley N. and Calatrava-Requena J. (2005). Designing policy for reducing the off-farm effects of soil erosion using Choice Experiments. Journal of Agricultural Economics, 56: 80-96.

Colombo, S., Calatrava-Requena J. and Hanley N. (2006): Analysing the social benefit of soil conservation measures using stated preference methods. Ecological Economics, 58(4): 850-861

Esyrce 2009. Encuesta sobre superficie y rendimientos de los cultivos. Disponible en: https://www.mapa.gob.es/es/estadistica/temas/estadisticas-agrarias/boletin2009_tcm30-122321.pdf

Esyrce 2019. Encuesta sobre superficie y rendimientos de los cultivos. Disponible en: https://www.mapa.gob.es/es/estadistica/temas/estadisticas-agrarias/agricultura/esyrce

EU (2021). List of potential agricultural practices that eco-schemes could support. Disponible en: https://ec.europa.eu/info/sites/info/files/food-farming-fisheries/key_policies/documents/factsheetagri-practices-under-ecoscheme_en.pdf

Espejo-Pérez, A. J., Rodríguez-Lizana, A., Ordóñez, R. \& Giráldez, J. V. (2013). Soil Loss and Runoff Reduction in Olive-Tree Dry-Farming with Cover Crops. Soil Sci. Soc. Am. J. 77, 2140.

Gómez, J. A. Campos, M. Guzman, G. Castillo-Llanque, F, Vanwalleghem T. Lora A. Giraldez, J. (2017). Soil erosion control, plant diversity, and arthropod communities under heterogeneous cover crops in an olive orchard. Environ. Sci. Pollut. Res. 1-13 (2017).

MAPA (2021). La aplicación de la condicionalidad reforzada en el marco de los planes estratégicos de la pac post 2020 (reglamento PEPAC). Disponible en: https://www.mapa.gob.es/es/pac/post2020/aplicaciondelacondicionalidadreforzadaenlosplanesestrategicosdelapac_tcm30-537081.pdf

Márquez-García, F., Sánchez-González, E.J., Castro-García, S. and Ordoñez-Fernández, R. (2013). Improvement of soil carbon sink by cover crops in olive orchards under semiarid conditions. Influence of the type of soil and weed. Spanish Journal of Agricultural Research, 11(2):335-346.

Nieto O.M., Castro J., Fernández E., Smith P. (2010) Simulation of soil organic carbon stocks in a Mediterranean olive grove under different soil management systems using the RothC model. Soil Use Management, 26: 118-125.

Parras-Alcántara, L., Lozano-García, B., Keesstra, S., Cerdá, A. \& Brevik, E. C. (2016). Long-term effects of soil management on ecosystem services and soil loss estimation in olive grove top soils. Science Total Environment. 571: 498-506.

Rodríguez-Entrena M., Colombo, S. and Arriaza M. (2017). Is landscape attractiveness a driver of the rural economy? The case of a pathway restoration in olive groves. Land Use Policy, 65: 164-175. 